\title{
Lenguaje y pensamiento en el desarrollo: Un nuevo modelo teórico
}

\author{
Miguel Pérez Pereira
}

Universidad de Santiago de Compostela.

El objeto del presente trabajo no es otro que el de realizar una revisión crítica de los estudios que versan sobre las relaciones existentes entre lenguaje y pensamiento en el desarrollo. Dicha revisión pretende sentar las bases de un nuevo modelo teórico más adecuado para representar las relaciones entre lenguaje y pensamiento. La necesidad de un modelo teórico tal surge, por una parte, de la convicción de que las teorías actuales existentes son incapaces de integrar los datos y conocimientos aportados por las investigaciones empiricas, $y$, por otra parte, de que la cantidad de datos y conocimientos aportados por estos trabajos empíricos - muy numerosos en los últimos quince años - posibilitan la elaboración de esta nueva teoría más capaz, integradora y adecuada.

\section{TEORIAS EXISTENTES}

A continuación, veremos las teorías más importantes sobre el tema.

I. Los autores enmarcados en la corriente conductista, tanto si sostienen la existencia de respuestas mediadoras (Osgood, Suci y Tannembaum, 1957; Mowrer, 1954; Reiss, 1969) como si no (Skinner, 1957) han adoptado una posición que lleva a considerar como esencialmente idénticos lenguaje y pensamiento. En definitiva, ambos procesos responden a las leyes que rigen los fenómenos del condicionamiento clásico o del operante, y consideran al lenguaje y al pensamiento como conductas externas y nunca como saberes estructurados internos, coherentemente con el antimentalismo de estos autores. Entre autores más recientes que Watson (1925), la diferencia fundamental entre lenguaje y pensamiento es que este último carece del componente motor externo que caracteriza al habla (Maltzman, 1955). De cualquier forma, el lenguaje es, según este mismo autor, una de las manifestaciones del pensamiento. Por consiguiente, se puede afirmar que los autores situados en la órbita del conductismo y neoconductismo sostiene una posición de identidad fundamental de habla y pensamiento, y de reducción de ambos a 
los principios que rigen los fenómenos del condicionamiento.

2. Whorf es quizá el represante más destacado de lo que se denomina la whipótesis del relativismo y determinismo lingüísticos». Sustancialmente viene a decir que el lenguaje que hablan los individuos condiciona su forma de comprender el mundo y su forma de pensar, por tanto. Dado que existen lenguas de gran diversidad (léxica y gramatical) entre sí, las formas de pensar y las concepciones del mundo de los hablantes de esas lenguas también difieren. Whorf trató de fundamentar empíricamente estas ideas con sus estudios de la lengua y la cultura de los indios hopi comparadas con las de los hablantes de las lenguas SAE (Standard Average European) (Whorf, 1956).

Diversos autores han criticado su vaguedad y escasa precisión en la definición de los términos, lo cual hace difíciles los estudios de comprobación de la hipótesis (Brown, 1958; Slobin, 1971; Fishman, 1960). Si bien ha habido intentos de sistematizar y concretizar las implicaciones de la hipótesis whorfiana por parte de estos dos últimos autores.

Los relativamente escasos estudios que pretendieron confirmar la hipótesis del relativismo y determinismo lingüístico en aspectos concretos y definidos han llevado a resultados en general desalentadores para los que defienden una fórmula fuerte de esta hipótesis (Cf. Brown y Lenneberg, I 95 4; Carroll y Casagrande, 1958...). Hoy en dia si tiende a aceptarse que la existencia de ciertas palabras y ciertos artificios gramaticales hacen más fácil la expresión de ciertas ideas en unos idiomas que en otros. Esta concepción constituye la fórmula débil de la hipótesis, lo cual es muy diferente a afirmar una determinación del pensamiento por el lenguaje de una manera absoluta y generalizada.

Una postura que, con un enfoque diferente, también ha sostenido la idea de la determinación del pensamiento y la conducta por el lenguaje es la de psicólogos soviéticos como Vygotsky (1934), y más claramente Luria (1959, 1961), y Luria y Yudovich (1956). Toda la teorización acerca del papel regulador del lenguaje sobre el desarrollo y sobre el comportamiento se inscribirá en esta línea, que ha sido criticada desde posiciones cognitivas (Flavell, 1977; Bronckart, 1973, 1977).

Igualmente se podría incluir en esta misma línea la obra de Bruner (1964) y de Bruner y otros ( 1966 ), para quienes la interiorización del lenguaje permite el inicio de una nueva forma de representación del mundo (la "simbólica»), que será mucho más evolucionada que las anteriores (representación "enactiva» e «icónica", basadas en la acción y la percepción, respectivamente), y permitirá superar sus limitaciones.

3. Una tercera posición sostiene que el lenguaje es enteramente independiente del pensamiento u otros aspectos del desarrollo, $y$, por tanto, no existe ninguna influencia de éstos sobre aquél en su desarrollo. Chomsky es el iniciador de esta corriente en la psicolingüística contemporánea. Según él, la adquisición del lenguaje se realiza gracias a la existencia de un LAD (Languaje Acquisition Devi$c e=$ Dispositivo para la adquisición del lenguaje) innato en la especie humana.

Al propio tiempo, Chomsky critica con dureza los intentos de explicar el aprendizaje de algo tan complejo como el lenguaje, apelando a los principios del aprendizaje S-R que operan sobre un corpus lingüístico limitado (Chomsky, 1959, 1965, 1975, etc.). La rapidez de este aprendizaje, según Chomsky, invalida la aplicación de dichos principios (cf. también Miller, Galanter y Pribram, I960). McNeill, quien se ha dedicado al estudio de la adquisición del lenguaje, sostuvo estas mismas tesis (McNeill, I 966).

4. Piaget (1954, 1967, 1946) y otros autores influidos por su teoría y/o por la teoria de la semántica generativa (Brown, 1973; Schlesinger, 1971...) han sostenido la idea de que el desarrollo lingüístico sigue los pasos marcados por el desarrollo intelectual, que aquél es feudatario y depende de éste.

Piaget demostró cómo el lenguaje no es, sino, una forma de representación, juntamente con otras formas como el juego simbólico, la imitación diferida, la imagen mental. Y que dicha capacidad de 
representar o función simbólica, que aparece sobre los dieciocho ó veinticuatro meses, hunde sus raíces en el período anterior de la inteligencia sensorio-motriz (Piaget, 1946). Por tanto, el pensamiento es anterior al lenguaje.

Piaget arguye que hay datos que indican que más adelante, en el desarrollo, el aprendizaje de formas lingüisticas no lleva a un aprendizaje de las operaciones mentales que se suponen relacionadas, lo cual contradice la hipótesis del determinismo linguístico contra la que combatió Piaget inicialmente. Además, la aparición de ciertas formas lingüísticas en el repertorio del niño exige la consecución previa de las nociones u operaciones mentales en que descansan.

Más adelante, Piaget (prólogo a Ferreiro, 1971 ) ha variado su posición, y en v́ez de sostener la hipótesis del determinismo cognitivo, ha pasado a defender una en la que se defiende la idea de que tanto el lenguaje como el pensamiento dependen de un factor general de organización del organismo, tal como expresa el gráfico.

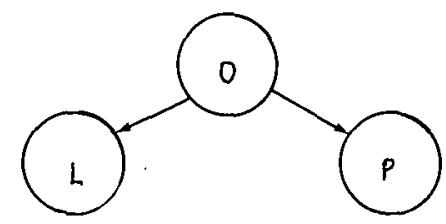

Donde:

$\mathrm{O}=$ Factor general de organización.

$\mathrm{L}=$ Lenguaje.

$\mathrm{P}=$ Pensamiento.

Otros autores han defendido también una hipótesis de determinismo cognitivo (Olson, 1970; Bever, 1970). Asi, Bever (1970) sostiene que el sistema de percepción del niño restringe las estructuras gramaticales que en cada momento del desarrollo, puede aprender.

5. Recientemente ha surgido una hipótesis que pretende superar las posturas extremas que afirman la primacía de uno de los dos polos en la polémica de las relaciones entre lenguaje y pensamiento. Según la hipótesis del determinismo cognitivo sólo tendría que aprender a proyec- tar las estructuras y conceptos cognitivos en formas lingüísticas apropiadas, lo cual se haría casi automáticamente una vez que se halla en posesión de las estructuras cognitivas correspondientes. Y según la hipótesis del determinismo lingüístico -que Schlesinger (1977) denomina del «imput lingüístico»-, el niño adquiere los conceptos por vía de la experiencia lingüística.

La hipótesis alternativa es denominada interaccionista (Bowerman, 1976), y los autores que la comparten diferencian claramente entre estructuras del conocimiento y estructuras lingüísticas (cosa que no siempre se ha hecho), y mantienen que ciertos aspectos del desarrollo lingúístico dependen de ciertos aspectos del desarrollo intelectual, pero también que el lenguaje se desarrolla autónomamente en otros aspectos y que puede influir en la construcción y más clara delimitación de las categorías cognitivas. Tal como afirma Bowerman (1976: I 12) «hay muchas relaciones posibles entre lenguaje y formación de conceptos dependiendo, a la vez, de las clases de conceptos envueltos y del tipo de input proviston. Otros autores que defienden esta posición interaccionista en el tratamiento de las relaciones entre el lenguaje y el pensamiento son Dore (1975), Schlesinger (1977), Zinkin (1976), Wells (1974), Bloom (1973), Karmiloff-Smith (1979), Mayor (1977), etcétera.

Una consecuencia que se deriva de tal posición es que las categorías cognitivas y las lingüísticas no se desarrollan en una correspondencia uno a uno, y que, por tanto, la existencia de determinadas estructuras conceptuales o categorias cognitivas no implica necesariamente que exista su contrapartida a nivel lingüístico y viceversa. Por tanto, pueden existir desajustes y faltas de correspondencia entre uno y otro nivel.

\section{CONTRASTACION EMPIRICA DE LAS TEORIAS}

Tal como vamos a ver, ninguna de las teorías expuestas es capaz de integrar 
completamente los datos aportados por las numerosas investigaciones realizadas sobre el tema últimamente, pues si bien la concepción interaccionista, como veremos, parece más adecuada, sin embargo, se encuentra formulada de una manera todavia muy primitiva.

La hipótesis reduccionista que tiende a identificar lenguaje y pensamiento, y que apela para explicar el aprendizaje de la lengua a principios como la repetición, el reforzamiento o la imitación, difícilmente podría dar cuenta del hecho de que niños afectados de disartría congénita puedan llegar a poseer competencia lingüistica (Lenneberg, i 962; Goodenough-Trepagnier, 1978). Esta concepción adolece, pues, de una manifiesta debilidad para explicar los fenómenos relativos al lenguaje y el pensamiento. Por otra parte, han sido ya contundentemente expuestas en la literatura psicológica las críticas al paradigma S-R (conductismo y neoconductismo), que se revela incapaz de describir y explicar los procesos más complejos caracteristicos del hombre (formación de conceptos, solución de problemas, lenguaje, razonamiento, memoria, etc.).

La hipótesis del desarrollo independiente del lenguaje, en su forma extrema, no puede dar una explicación adecuada a los resultados aportados por numerosos autores que han hallado un paralelismo entre el desarrollo de estas nociones conceptuales y operaciones lógicas y el desarrollo de ciertas estructuras lingüísticas a partir de los dos años (cf., por ejemplo, los estudios sobre la conservación de cantidades y seriación y su expresión lingüística, sobre las relaciones temporales y sobre la voz pasiva y sus soportes cognitivos: Sinclair, 1967; Ferreiro, 1971; Lempert, 1978; Ehri, 1976, etc.).

Igualmente, se revela incapaz de dar una explicación convincente del hecho de que para que aparezca el primer lenguaje (combinatorio) inicial del niño debe haber alcanzado un cierto nivel en el desarrollo intelectual sensoriomotriz y comunicacional prelingüístico. En una serie de investigaciones realizadas, se ha comprobado que los niños, antes de la aparición de las primeras palabras, deben ha- ber adquirido ciertas cotas en su desarrollo intelectual; cotas que se logran al final del período sensóriomotriz (cf. para una revisión completa del tema Corrigan, I 979 y Pérez Pereira, I98I).

Pero estas condiciones, tal y como la propia Sinclair apunta (Sinclair, 1971:119), no parecen ser suficientes para la aparición del lenguaje combinatorio. Parece ser que se requieren también ciertos logros en otras áreas antes de que el niño emplee de una forma combinada dos palabras. Esta idea surge de ciertas excepciones que se han encontrado a estas normas (Ingram, 1978), así como de las múltiples oscilaciones halladas en diferentes investigaciones acerca del momento en que aparecen las primeras palabras y el momento en que se comienzan a combinar en relación al estadio del desarrollo intelectual en que se halla el niño (Pérez Pereira, 1981).

Además de estos prerrequisitos cognitivos, que deben llegar a tener una representación semántica, el niño debe lograr también un cierto nivel en lo que a su competencia comunicativa se refiere. Bruner (1975, 1978, etc.) y R. A. Clark (1 978) han descubierto ejemplos de esa capacidad para comunicarse por medios no verbales en fenómenos como la posibilidad de establecer una «atención compartida», o una "acción conjunta», o en cierto tipo de conductas reguladas por reglas: juego del "cu-cu», dar y tomar objetos, empleo de gestos con inténción comunicativa... E incluso se ha llegado a establecer qué gesto y palabra se usan unidos en un comienzo, lo cual favorece el aprendizaje de los significados de las palabras y su uso en sustitución de los gestos como forma preponderante de comunicación (Nokony, 1978; Ninio y Bruner, 1978 ).

Otro tipo de requisitos que parecen ser previos a la aparición del lenguaje se relacionan con los aspectos más formales del lenguaje (fonología, morfología y sintaxis). Obviamente es necesario un cierto desarrollo fonológico en el niño (Kaplan y Kaplan, 1970) que desemboque en las primeras palabras reconocibles partiendo de «morfemas sensoriomotrices» primiti- 
vos y de "gestos vocales" Carter, 1975 , 1978, 1979; Nokony, 1978). Igualmente se ha apreciado la constitución de "formas fonéticamente consistentes» y de «dispositivos presintácticos», que anteceden a la aparición de las designaciones léxicas (palabras) y a las primeras combinaciones sintácticas, respectivamente (Dore, Franklin, Miller y Ramer, 1976).

En definitiva, que para que aparezca el lenguaje es necesario que se dé un cierto nivel de desarrollo en las áreas de lo cognitivo, lo comunicacional, y lo formal-verbal. Es justamente su confluencia la que posibilita el inicio de la competencia lingüistica. Sólo cuando el niño es capaz de integrar estos logros aparece el lenguaje, es decir, la capacidad de expresar unos contenidos semánticos (que reflejan contenidos conceptuales) en formas lingüisticas que se ajustan a determinadas reglas gramaticales que se usan adecuadamente teniendo en cuenta el contexto y con el fin de alcanzar determinados propósitos ejerciendo sobre otros el efecto adecuado para ello.

La experiencia cognitiva y comunicacional y de verbalización anterior parece, así pues, fundamental para la aparición del lenguaje en el niño. Pero ha de quedar bien claro que el lenguaje, desde el momento de su institución se constituye como algo cualitativamente diferente de los logros anteriores en cada uno de estos planos.

Estos y otros hechos revelan que el desarrollo estrictamente lingüistico no puede ser ajeno a la influencia de otros aspectos del desarrollo, como el cognitivo y el social.

No obstante, los paralelismos hallados entre evolución de ciertas nociones y operaciones mentales y la evolución de ciertos aspectos del contenido, forma, o uso del lenguaje, aquellos no pueden ser interpretados necesariamente como la evidencia de que existe una relación causal entre pensamiento y lenguaje, siendo éste una traducción mecánica del primero (cf. Dore, 1975, y Monitz-Rodgon, 1976, para una crítica a este salto en el razonamiento).

Por otra parte, existen una serie de resultados de investigaciones que no son ajustables a las interpretaciones de quienes defienden la hipótesis del determinismo cognitivo. Entre éstos se hallan los de quienes encontraron que aparecen formas lingüisticas antes de la consolidación de las estructuras del pensamiento que le corresponden (cf. por ejemplo, Moore y Harris, sobre la voz pasiva y la reversibilidad operatoria). Estos casos, en definitiva, son exponente de la relativa independencia del desarrollo lingüistico y de la inexistencia de una relación tan inexorable entre el pensamiento y el lenguaje como pretenden los psicólogos defensores de la hipótesis cognoscitiva.

En esta misma linea de interpretación se ubican los resultados de aquellas investigaciones en las que se apreció que no todos los sujetos que adquirieron una noción determinada o unas operaciones lógicas alcanzan de inmediato las formas lingüisticas que supuestamente le corresponden (cf. Slobin, I 973; Ammon y Slobin, 1979, e incluso los resultados de Sinclair, 1967). Algunos autores interpretan este retardo en la aparición de formas lingüísticas que expresan conceptos ya adquiridos como la manifestación de la intervención de factores estrictamente lingüísticos en la adquisición de tales formas. De tal manera que hay lenguas que, por expresar de una forma más compleja que otras ciertas relaciones, hacen más difícil su adquisición por los niños. Esta mayor facilidad o dificultad en la adquisición está, indudablemente, influenciada por las claves morfológicas, fonológicas o sintácticas que aportan diferencialmente las distintas lenguas a los niños. Smith (1980) en un estudio sobre la comprensión de las relaciones temporales emplea pautas de interpretación semejantes para explicar la evolución en su adquisición en niños franceses e italianos, por una parte, e ingleses, por otra, habida cuenta de la mayor complejidad de la expresión de éstas en francés e italiano. Pero hechos como éste se dan también en el interior de una misma lengua: recordemos, por ejemplo, los estudios de Lempert (1978), Sinclair y Ferreiro (1970) y Ferreiro (1971) sobre la adquisición de la pasiva y la 
expresión de las relaciones temporales. En ellos se apreció que las oraciones que emplean verbos reversibles (lavar, etc.), que presentan claves semánticas y sintácticas que facilitan la tarea, son comprendidas y usadas antes que aquellas oraciones con verbos como «seguir» y "golpear», que no aportan dichas claves.

Otros datos que vienen a turbar a los defensores del determinismo cognitivo muestran que el entrenamiento verbal, es decir, el aprendizaje de ciertas formas lingüisticas, ejerce cierto efecto favorecedor sobre el desarrollo de estructuras operatorias y nociones conceptuales. De las experiencias de Heber (1977) sobre la seriación y de Rattan (1974) y Sinclair (1967) sobre la conservación de cantidades y la seriación se pueden extraer datos que apoyan esta interpretación.

Igualmente los estudios realizados con niños ciegos de nacimiento (Hatwell, I 966; Cromer, 1973) apuntan que el lenguaje parece jugar un papel favorecedor del desarrollo intelectual y de compensación de la privación sensorial, bajo ciertas condiciones. Con esto parece confirmarse la propuesta de Blank (1974) de que el lenguaje ejerce una acción compensadora en el razonamiento cuando falta la información visual.

Otras investigaciones muestran que el lenguaje (el empleo de unos términos u otros), así como la manera en que se dispone el material de las tareas que deben realizar los niños, intervienen en los resultados que obtienen éstos en las pruebas operatorias (cf. McGarrigle y otros, 1978; Rommetveit, 1978; Shipley, 1975). Ciertos términos dificultan la comprensión de las consignas o crean un efecto de "halo" que entorpece la correcta realización de las tareas.

Schlesinger (1977), por otra parte, ha apuntado que el lenguaje (distinción entre nombres propios sin artículo, y comunes precedidos de artículo) puede dirigir la atención del niño para interpretar acontecimientos de su ambiente y, en definitiva, ser el causante de la aparición de nuevos conceptos mentales en el niño (distinción entre seres humanos y no humanos).
En definitiva, que la hipótesis del determinismo cognitivo, que había sido apoyada por ciertos datos empíricos, tampoco está libre del efecto devastador de ciertos datos, y se manifiesta también como una teoría inapropiada para reflejar y explicar las relaciones entre lenguaje y pensamiento. Aun a pesar de las evidencias que apuntan a la influencia del lenguaje en el desarrollo cognitivo del niño, y a la influencia de claves estrictamente lingüísticas en el proceso de adquisición del lenguaje, existen también una serie de estudios que muestran las limitaciones de un enfoque determinista lingüístico.

Entre éstos se hallan los desarrollados con unos niños sordos (Furth, 1966; Furth y Youniss, I97I, Oléron y Herren, I95 I, etcétera), en los que se comprobó que es posible un desarrollo considerable del pensamiento sin necesidad de que exista lenguaje. Por otra parte, el retraso en el desarrollo que se aprecia en los sujetos sordos no es seguro que se deba exclusivamente a la carencia lingüística, como pretende Oléron (1972).

En experiencias realizadas sobre el tema se aprecia que el entrenamiento lingüistico no es suficiente para que se dé desarrollo operatorio; de lo contrario todos los sujetos que llegan a usar correctamente términos y estructuras gramaticales que se emplean para la expresión de ciertas nociones deberían también haber alcanzado éstas, cosa que no es en absoluto real (Sinclair, 1967; Ehri, 1976, por ejemplo).

Otros autores han mostrado la influencia de aspectos cognitivos sobre los lingüísticos. Por ejemplo, Johnson y Scholnick (1979), Delval y Carretero (1978), Beilin (1975) apreciaron un efecto de la estructura lógica sobre el razonamiento y la memorización con materiales verbales.

En ocasiones se ha constatado también que en el aprendizaje de ciertas formas verbales se emplean claves contextuales, tal como ocurre con las expresiones temporales o en el aprendizaje de términos comparativos y adjetivos dimensionales (Klatz y otros, 1973; Donaldson y McGarrigle, 1974; Schwan, 1980). Claves 
que son aportadas por los sistemas perceptual y cognitivo.

\section{UN NUEVO MODELO TEORICO}

Parece, pues, fundamental sostener que ninguno de los modelos propuestos es adecuado para dar cuenta de las relaciones existentes entre pensamiento y lenguaje en el desarrollo, y que se hace preciso elaborar una teoría al respecto que sea menos parcial que las existentes. Al propio tiempo, el cúmulo de datos recogidos por las numerosas investigaciones realizadas permiten afrontar con ciertas garantías la tarea, y esto independientemente de las insuficiencias metodológicas y de procedimiento en que incurren algunas de ellas (muchas de las realizadas sobre las relaciones entre egocentrismo y desarrollo pragmático del lenguaje descuellan por ese motivo).

Un punto importante del modelo que se propone es el de adoptar una caracterización amplia de lo que es la competencia lingüística. Tal caracterización se apoya en los estudios ya citados acerca de los prerrequisitos necesarios para la adquisición del lenguaje. En lo que a la psicolingüistica evolutiva se refiere las caracterizaciones que de la competencia lingüística realizan las teorías del lenguaje (señaladamente la gramática generativa, la semántica generativa y "caso grammar», y la teoria de los actos de habla) parecen parcializadoras y no integradoras de las múltiples habilidades que el niño debe adquirir para llegar a ser un hablante fluente de un idioma. En este sentido, ya Hymes (1971) reveló la necesidad de integrar la capacidad para hacer un uso del lenguaje adecuado al contexto y a la expresión de intenciones y consecución de efectos deseados en lo que las teorías generativistas denominan "competencia lingüística» del hablante. Bloom y Lahey (1978) han desarrollado esta nueva dirección en la caracterización de la competencia lingüistica y su adquisición, y la han arropado con un soporte empirico.

Según ésta, el individuo aprende en su desarrollo a usar y comprender el lenguaje en relación (o como una representación de) las ideas o conceptos mentales que ha formado a lo largo de su experiencia. Tenemos, pues, un rasgo esencial del lenguaje; a saber que se usa para expresar ciertos contenidos mentales. Pero; y dado que el lenguaje es algo formal (Chomsky) y convencional (De Saussure), es necesario llegar a aprender las reglas que lo rigen. Por supuesto, éste es un conocimiento no explicito. Por tanto, es necesario llegar a dominar los aspectos formales del lenguaje. Pero, además, para ser un hablante de un idioma se precisa saber hacer un uso adecuado de él en relación al contexto lingüístico y extralingüístico y saber usarlo apropiadamente para la expresión de intenciones (órdenes, prohibiciones, preguntas, etc.), y para la consecución de los resultados que se pretenden. Este último aspecto de la competencia lingüística encarna especialmente lo que de medio de comunicación posee el lenguaje.

Así pues, el lenguaje presenta tres dimensiones fundamentales: la del contenido, la de la forma y la del uso.

El contenido del lenguaje es su significado o semántica, es decir, la representación lingüística de lo que una persona conoce acerca del mundo. Por tanto, es el aspecto del lenguaje que más directamente se relaciona con el desarrollo cognitivo del niño (Zachry, 1978, expone una idea semejante).

La forma del lenguaje es describible de diferentes maneras no antagónicas, sino complementarias. Puede hacerse en términos de las unidades de sonidos, lo que hace la fonología; de las unidades mínimas dotadas de significación, aspecto estudiado por la morfología; y de la forma en que las unidades con significación se combinan unas con otras para formar oraciones, que constituye el objeto de la sintaxis. Este aspecto de la competencia lingüística incumbe, sobre todo, al desarrollo gramatical y fonológico.

El uso del lenguaje presenta dos aspectos fundamentales. El primero tiene que ver con los fines o funciones del lenguaje, los motivos por los que la gente habla: 
constituye la expresión de intenciones o «fuerza ilocucionaria». La segunda tiene que ver con la influencia del contexto, que afecta a la manera como los individuos comprenden el lenguaje y escogen formas lingüísticas diferentes para alcanzar sus fines. Este aspecto de la competencia lingüística se relaciona especialmente con el desarrollo comunicacional y social cognitivo.

La competencia lingüística puede ser definida entonces como una integración de contenido, forma, y uso. Lo que implica la capacidad de expresar y comprender contenidos en formas lingüisticas adecuadas al contexto y para el logro de ciertas intenciones. Con el desarrollo de la competencia lingüistica del niño, éste va siendo capaz de expresar más contenidos, y de hacerlo de una manera más adecuada al contexto, empleando para ello un repertorio de formas lingüísticas cada vez mayor. Lo cual le capacita para expresar un mismo contenido con formas variadas y diferencialmente apropiadas al contexto.

Un segundo punto central del modelo propuesto lo constituye el de sostener una diferenciación neta entre el plano del lenguaje y el plano cognitivo, de tal manera que en él no hay cabida para la consideración de una traducción mecánica de los logros en uno de estos planos al otro, cualquiera que éste sea, con lo cual este modelo se diferencia netamente de los que aquí he denominado determinismo cognitivo y determinismo lingüístico, y es acorde con los datos analizados. Cabe, pues, en él la posibilidad de que existan desajustes en el desarrollo entre uno y otro plano. Por otra parte, el presente modelo integra las aportaciones de autores soviéticos que hipotetizan la existencia de una serie de etapas entre el pensamiento en su forma pura inicial y el lenguaje externo desplegado (Akhutina, 1978; Leontiev, I969; Luria, 1975, 1979).

Otro rasgo característico del modelo propuesto lo constituye la utilización de la noción de estrategia. Recientemente se ha apelado a este concepto para explicar el proceso de adquisición del lenguaje (Cromer, 1976; E. V. Clark, 1973, 1977...).
Según éste, la adquisición del lenguaje en el niño se caracteriza por la adopción de hipótesis y estrategias progresivamente más adecuadas para el uso y la comprensión de las diferentes formas lingüísticàs. Inicialmente, las estrategias que adopta el niño serán erróneas o parciales, no considerando, por ejemplo, las diversas funciones que una misma forma puede cubrir (véase a este respecto el trabajo sobre los determinadores y la expresión de la referencia de Karmiloff-Smith, 1979), o el significado apropiado adulto de un término (E. V. Clark, 1973, I 977).

En la formación de estas estrategias intervienen tanto claves provinientes de los conocimientos y experiencia física y social que posee el sujeto, como del contexto sujeto a la interpretación que le da el niño, como claves estrictamente lingüisticas, pudiendo ser éstas fonológicas, morfológicas o sintácticas (cf. Karmiloff-Smith, 1979, 1978; Ammon y Slobin, 1979; Slobin, 1973; Sinclair y Ferreiro, I970; Ferreiro, 1971; Lempert, 1978; Bowerman, 1974; Bronckart, I 977; Wales, 1979; Johnston y Slobin, 1979; Schlesinger, 1977; Bever, 1970; E. V. Clark, 1973, 1977, etcétera).

Por tanto, si bien es cierto que no son los aspectos cognitivos los únicos que determinan el momento y el proceso de adquisición de formas lingüísticas, tampoco se puede considerar su desarrollo independiente de estos aspectos. Es decir, que en el proceso de adquisición del lenguaje el niño va utilizando e integrando claves que provienen tanto de su experiencia física y social como de su experiencia lingüística.

Esta concepción permitiría explicar tanto la variación interindividual como la intraindividual en el desarrollo lingüístico (Bloom y Lahey, 1978; Schlesinger, 1977, han resaltado esta característica). Unos niños le darian más importancia a ciertas claves y otros a otras. De igual forma un mismo niño emplearia claves fundamentalmente cognitivas, por ejemplo, en el aprendizaje de un aspecto del lenguaje, y fundamentalmente lingüística en el aprendizaje de otro. $E$ incluso, permite esta concepción tener en cuenta 
los cambios que en el aprendizaje de una misma forma lingüística se aprecian en el niño desde el empleo de una a otra clave.

El empleo de mayor número de claves actuantes ocurrirá sobre todo en estados más evolucionados del desarrollo, en que las estrategias serán más ajustadas al modelo adulto; mientras que al comienzo el niño no podrá tener en cuenta todas ellas, siendo su hipótesis parcial, limitada e incorrecta (cf. Karmiloff-Smith, 1979; E. V. Clark, 1977).

Por otra parte, el modelo propuesto permite representar adecuadamente el hecho de que las estrategias empleadas para solucionar problemas, sean éstos lingüísticos, físicos o de otro tipo, sean semejantes (Karmiloff-Smith e Inhelder, 1975), así como la existencia de una cierta sincronía en el desarrollo. El factor que Piaget denominó (Piaget, 197I) «mecanismo regulador y organizador» representaria esas capacidades generales procesuales y de formación de estrategias que se han de emplear posteriormente para la solución de los problemas que se planteen en los diferentes planos (cognitivo, lingüístico y social).

Pero al propio tiempo, el modelo propuesto permite representar también un hecho incontestable. $Y$ es que esa capacidad operativa general no surge de la nada, sino que se desarrolla en base a la experiencia del niño en los tres planos representados (cognitivo, lingüístico y social). La influencia de estos tres planos sobre el desarrollo de ese «mecanismo regulador y organizador» se representa en el gráfico que sigue mediante las flechas de doble dirección.

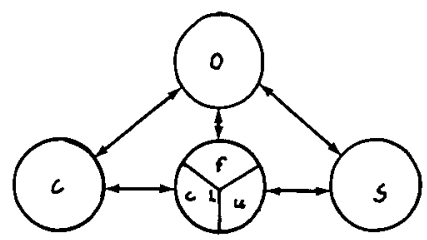

Donde:

$\mathrm{O}=$ Mecanismos de organización y regulación generales.

$\mathrm{C}=$ Plano del desarrollo cognitivo.

$\mathrm{S}=$ Plano del desarrollo social-comunicacional.

$\mathbf{L}=$ Plano del desarrollo lingüístico, con sus tres aspectos de contenido (c), forma (f) y uso (u).

Las flechas de doble dirección indican interacción.
El desarrollo en uno de estos planos afectaría también, por tanto, indirectamente al desarrollo de los otros dos. Con esto integro también la incisiva crítica que Bates y otros (1977) realizaron a quienes consideran que el desarrollo de esa "capacidad operativa general» se realiza sólo, o fundamentalmente sólo, en base a la experiencia física y social (base del desarrollo cognitivo en la teoría piagetiana) del niño, y que en la experiencia física se halla la fuente y manifestación principal y de esencia de las capacidades cognitivas generales del niño. En esta concepción, criticada por Bates y col., la esfera del lenguaje no sería más que una manifestación secundaria de aquéllas, y no intervendría para nada en su desarrollo.

En concordancia ccn este modelo, el lenguaje es un área que presenta problemas al niño y que exige de éste la puesta en actividad de mecanismos intelectuales que los resuelvan. Esto, obviamente, debe estimular, a su vez, el propio desarrollo de ese mecanismo regulador y organizador, lo cual repercutirá también sobre el desarrollo en el plano cognitivo. En este sentido, las correlaciones entre el desarrollo intelectual y el desarrollo lingüístico se deberían buscar no tanto entre conceptos cognitivos concretos y formas lingüísticas que los expresan (sin negar su existencia), como en el uso de estrategias, hipótesis, modelos heurísticos, etc., similares.

A medida que progresa el desarrollo, la influencia del lenguaje sobre la evolución intelectual es mayor. $Y$ esto se plasma de dos maneras. De una manera indirecta al incidir sobre el «mecanismo general de regulación y organización” del organismo, en tanto que afecta a las capacidades generales de procesamiento y formación de estrategias e hipótesis, lo que, en definitiva, afectará al desarrollo cognitivo. $Y$ de una manera directa, en tanto es posible cada vez más una transmisión verbal de conocimientos, por vía de lo que Ausubel (1968) ha denominado aprendizaje verbal significativo.

Además de representar adecuadamente datos concernientes al desarrollo normal, el presente modelo es capaz también de 
integrar fenómenos como el de los afásicos, que se caracterizan por poseer un razonamiento normal, pero un lenguaje muy desestructurado, al ser las estructuras del lenguaje y del pensamiento diferenciadas. $\mathrm{O}$, por la misma razón, permite dar cuenta del caso de los niños sordomudos, quienes a pesar de no poseer lenguaje presentan cierto desarrollo del pensamiento. Por otra parte, en el caso de los niños ciegos, ocurriria que éstos al estar privados de su experiencia sensorial, se apoyarán, para su desarrollo cognitivo $\mathrm{y}$ de los mecanismos reguladores y organizadores generales, en la experiencia lingüistica (y social). Se produce, por tanto, un fenómeno de sobrecompensación en los casos de anomalías en el desarrollo. Idea frecuentemente aceptada en la bibliografia soviética (Tsvétkova, I977), y que descansa en la teoría de los "sistemas funcionales» elaborada por Anojin $y$ Vygotsky.

Incluso este modelo permite explicar e integrar los datos relativos a casos de desajustes o "decalages» en el desarrollo de la competencia lingüística. Entre éstos se hallarían casos extremos como los de los niños afectados de anartría, que pueden poseer significados semánticos -es decir, hay un desarrollo relativo del aspecto de contenido-, pero es incapaz de expresarlos en forma lingüística (por incapacidad articulatoria en este caso); o los casos de los diferentes tipos de afasias.

Este modelo se podría enmarcar dentro de lo que se ha denominado posición interaccionista, si bien lo desarrolla $y$ matiza. Recoge datos e interpretaciones aisladas aportadas por varios investigadores, integrándolas en un marco más amplio.

De este modelo se deduce una recomendación general para las diversas investigaciones por realizar. Y es que éstas han de plantearse de una manera tremendamente concreta, evitando las generalidades. Para ello es necesario delimitar perfectamente la habilidad lingüistica que se desea estudiar, sus posibles funciones (KarmiloffSmith, 1979), los aspectos lingüísticos implicados en ella (contenido, forma, uso) y las relaciones concretas que puede presentar con otros planos del desarrollo (cognitivo y social). En este sentido entiendo que el modelo que propongo puede ejercer una fructífera función de guía de futuras investigaciones.

Por supuesto, este modelo general requiere ser afianzado y rellenado de contenido mediante investigaciones empiricas concretas. en este sentido es tentativo, si bien los datos existentes parecen apoyarlo.

\section{Referencias}

Akhutina, T. U. (1978): "The role of inner speech in the construction of an utterance». Soviet Psychology, I $978, \mathrm{XVI}, 3,3-30$.

AmMON, M. S., y SLOBIN, D. T. (I 979): "A cros-linguistic study of the processing of causative sentences». Cognition, 1979, 7 (1), 3-18.

Ausuber, D. P. (1968): Psicologia Educativa: Un punto de vista cognoscitivo. Trillas. México, 1976. Original: Educational Psycbology: A cognitive view. Holt, Rinehart and Winston, 1968.

Bates, E.; Benigni, L.; Bretherton, I.; Camaioni, L., y Volterra, V. (1977): «From gesture to the first word: on cognitive and social prerequisites". En Lewis, M., y Rosemblum, L. (eds.): Interaction, conversation and the development of language. Origins of behavior: Communication and language. John Wiley. N. Y., $1977,247^{-307 .}$

Hatwell, Y (1966): Privation sensorielle et intelligence. P. U. F. París, i966.

BEIL.IN, H. (1975): Studies in cognitive basis of language development. Academic Press. N. Y., 1975.

BEver, T. G. (1970): "The cognitive basis for linguistic structures". En Hayes, J. R. (ed.): Cognition and the development of language. John Wiley \& Sons. New York, 1970, 279-362.

Bloom, L. (1973): One word at a time: the use of single word utte nes efre $y$ tax. Mouton. La Haya, 1973.

BIOOM, L., y LAHEY, M. (1978): Language develoment and language iso der. John Wiley. N. Y., I978.

BOWERMAN, M.: (1974): «Learning the structure of causative verbs: A study in the relationship of cognitive, semantic and syntactic development». Papers and Reports on child language development, 1974, 8, $142-178$.

Bowerman, M. (1976): "Semantic factors in the acquisition of rules for word use and sentence constructions. En Morehead, D. M., y Morehead, A. E. (ed.): Normal and deficient child language. University Park Press. Baltimore, 1976. 
BronCKart, J. P. (1973): "The regulation role of speech: A cognitivist approach». Human Development, $1973,16(6), 417-439$.

Bronckart, J. P. (1977): "Adquisición del lenguaje y desarrollo cognitivo». En Bronckart, J. P., y otros: La génesis del lenguaje. Symposium de la Asociación Cientifica Francesa, 197s. Pablo del Río Editor. Madrid, 1978. Original: La gènese de la parole. P. U. F., 1977, 105-109.

Brown, R. (1958): Words and things. An introduction to language. The Free Press. N. Y., 1958.

Brown, R. (1973): A first language. George Allen and Unwin Ltd. London, 1973.

Brown, R. W., y Lenneberg, E. H. (1954): A study language an cognition Journal of Abnormal and Social Psychology, $1954,49,454-462$.

BRUNER, J. S. (1964: "The course of cognitive growth". American Psychologist, 1964, 19, 1-16.

BRUNER, J. S. (1975): "The autogenesis of speech acts". Journal of Child Language, 1975, 2 (1), I-19.

BRUNER, J. S. (1978): "On prelinguistic prerequisites of speech». En Campbell, R. N., y Smith, P. T., 1978, 199-214.

Bbruner, J. S.; Olver, R. R., y GreEnfIELD, R. M. (1966): Investigaciones sobre el desarrollo cognitivo. Pablo del Rio Editor. Madrid, 1980. Studies in cognitive growth, John Wiley and Sons. 1966.

CAMpbel.l., R. N., y SmiTh, P. T. (eds.) (1978): Recent advances in the psychology of language, vol. 1. Language development and mother-child interaction. Plenum presss. New York, 1978.

Carrol.t., J. B. (1964): Language and thought. Prentice-Hall. Englewood Cliffs, 1964.

CARrol.., J. B., y CASAGR ANDE, J. B. (I 958): Language structuring experience. En Rogers, S. (ed.), i 975 , págs. 187-208. Original en Maccoby, E. E.; Newcomb, T. M., y Hartley, E. L. (eds.) Readings in social psychology. Holt, t998.

CARTER, A. L. (1975): "The transformation of sensorimotor morphemes into words: A case study of the development of "more" and "mine"), Journal of Child Language, 1975, 2 (2), 233-290.

CARTER, A. L. (1978): From sensori-motor vocalizations to words: A case study of the evolution of attention directing communication in the second year. En Lock, A. (ed.), 1978, 309-349.

CARTER, A. L. ( 1979): "Prespeech meaning relations: An outline of one infant's sensorimotor morpheme development". En Fletcher, P., y Garman, M. (eds.): Studies in language acquisition. Cambridge Univ. Press. Cambridge, 1979, $71-92$.

Clark, E. U. (1973): What's in a word? On the child's acquisition of semantics in bis first language. En Moore, T. E., 1973, págs. 65-110.

Clark, E. U. (1977): "First language acquisition». En Morton, J., y Marshall, J. C. (eds.): Psycholinguistic series. Vol. I Developmental and patbological. Elek Press. Londres, 1977, I-73.

Cl.ARK, R. A. (1978): The transition from action to gesture. En Lock, A. (ed.), 1978, $231-257$.

CORRIGAN, R. (1979): «Cognitive correlates of languege: Differential criteria yield differential results». Child Development, 1979, so (3), 617-631.

Cromer, R. F. (1973): «Conservation by congenitally blind». The British Journal of Psychology, 1973, $64,241-250$.

CROMER, R. F. (1976): «Developmental strategies for language». En Hamilton, V., y Vernon, M. D. (eds.): - The development of cognitive processes. Academic Press. Londres y N. Y., 1976, $305-358$.

Chomsky, N. (1959): Recensión crítica de "Verbal Behavior», de B. F. Skinner. Convivium, 1973, 38, 65-105. Original en Language, 1959, 35 (1), 26-58.

Chomsky, N. (1965): Aspectos de la teoria de la sintaxis. Aguilar. Madrid, 1970. Original en MIT Press. 1965.

Chomsky, N. (1975): Reflexóes sobre a linguagem. Ediçoes 70. Lisboa, 1977. Original: Reflections on language. Pantheon Books, 1975.

Delval, J., y Carretero, M. (1978): “La adquisición de las conectivas proposicionales por los niños». En Delval, J. (comp.): Lecturas en psicologia del niño. Vol. 2. Alianza. Madrid, 1978, I78-1 94.

DONALDSON, M., y McGARRigle, J. (1974): "Some clues to the nature of semantic development". Journal of Child Language, 1974, I (2), 18 5-194.

DORE, J. (1979): «Holophrases, speech acts and language universals». Journal bi Language, i 975, 2,2 1-40.

DORE, J.; FRANkLIN, M.; MILI.ER, R., y RAMER, A. (1976): "Transitional phenomena in early language acquisition". Journal of Child Language, 1976,3 (1), $13-28$.

EHR!, L. C. (1976): "Comprehension and production of adjectives and seriation". Journal of Child Language, $1976,3(3), 369-384$.

FERREIRO, E. (1971): Les relations temporelles dans le langage de l'enfant (Preface de Jean Piaget). Droz, Ginebra, I971.

FishmaN, J. A. (1960): «A systematization of the Whorfian hypotheses». Bebavioral Science, 1960, 5, 323-339. Fl.avel.., J. H. (1977): Cognitive Development. Prentice-Hall, 1977.

FURTH, H. G. (1 966): Thinking without language. Psychological implications of deafness. The Free Press. N. Y., 1966.

FurTH, H. G., y YounIsS, J. (197I): "Formal operations and language, a comparison of deaf and hearing adolescentsm. International journal of psycbology, 1971, 6 (1), 49-64.

GOODENOUGH-TREPAGNIER, C. (1978): Language development of children without articulate speech. En Campbell, R. N., y Smith, P. T. (eds.), 1978, 1, $421-426$.

HEBER, M. (1977): "The influence of languaje training on seriation of $5-6$ yeards old children initially at different levels of descriptive competence». British Journal of Psychology, 1977,68 (1), 8 s-95.

HENLE, P (1958): Languaje thought and culture. University of Michigan Press, 1972. Primera edición, 1958.

Huxi.ey, R., e INGRAM, E. (eds.) (1971): Languaje acquisition: Models and methods. Academic Press. London y New York, 1971 . 
HYMES, D. (1971): Competence and perfomance in linguistic theory. En Huxley, R., e Ingram, E. (eds.), 1971, 3-24. INGRAM, D. (1978): “Language development during sensorimotor period». En Waterson, N, y Snow, C. (eds.). The development of communication: Social and progmatical factors in language acquisition. Wiley. Londres, 1978, 379-389.

Johnston, J. R., y SLOBIN, D. I. (1979): "The development of locative expressions in English, Italian, Servo-Croatian and Turkish. Journal o Child Language, 1979, 6, 529-545.

JOHNSON, J. W., y SCHOLNICK, E. K. (1979): "Does cognitive development predict semantic integration?". Child Development, 1979, 50 (1), 73-78.

Kaplan, E, y KAPLAN, G. (1971): «The prelinguistic child. En Eliot, J. (ed.). Human development and the cognitive processes. Holt, Rinehart \& Winston, 1971, 358-381.

KARMILOFF-SmITH, A. (1978): The interplay between syntax, semantics and phonology in language acquisition processes. En Campbell, R. N., y Smith, P. T., 1978, 1, 1-23.

Karmilof-Smith, A. (1979): A functional approacb to cbild languaje. A studie of determiners and reference. Cambridge University Press. 1979.

Karmiloff-Smith, A., e INHelder, B. (1975): "If yo want to get ahead, get a theory». Cognition, i975, 3, 195-212.

KLATZ, R. L.; CLARK, E. V., y MACKEN, M. (1973): «Asymmetries in the acquisition of polar adjectives: Linguistic or conceptual?». Journal of Exp. Child Psychology, 1973, 16 (1), 32-46.

LEMPERT, H. (1978): "Extrasyntactic factors affecting passive sentence comprehension by young children». Child Development, 1978, 49 (3), 694-699.

LENNEBERG, E. H. (1962): «Understanding language without ability to speak: a case report». Journal of Abnormal an social psychology, 1962, 65 (6), 419-425.

LEONTIYEV, A. A. (1969): «Inner speech and the processes of grammatical generation of utterances». Soviet Psychology, 1969, 7 (3), I I-16.

Lock, A. (ed.) (1978): Action, gesture, and symbol: the emergence of language. Academic Press. New York, 1978.

LURIA, A. R. (1959): "The directive function of speech in development and dissolution". Word, I959, 15, 341-392.

LuRiA, A. R. (1961): Lenguaje y Comportamiento. Fundamentos, Madrid, 1974. Original: The role of speech in regulation of normal and abnormal bebavior. Pergamon Press, 1961 .

Luria, A. R. (1975): Lenguaje y pensamiento. Ed. Fontanella. Barcelona, 1980. Original ruso, 1975.

Luria, A. R. (1979): Conciencia y lenguaje. Supervisión de E. D. Jonskaia. Pablo del Rio Editor. Madrid, I980. Original ruso, I979.

Luria, A. R., y YUdovich, F. IA. (1996): Lenguaje y desarrollo intelectual. Pablo del Río Editor. Madrid, 1978. Original en ruso, 1956.

Maltzman, I. (1955): "Thinking from a behavioristic point of view». Psychological Review, 1959, 62 (4), 275-286.

MATHIOT, M. (1977): "On the study of the relation of language to thought». Communication and Cognition, $1977,10(1), 77^{-82}$.

MAYOR, J, (1977): Comunicación y lenguaje. Universidad Internacional Menéndez Pelayo. Santander, 1977.

MCNeill, D. (1966): "The creation of language». Discovery, 1966, 27 (7), 34-38. Versión española en Luria y otros: Lenguaje y psiquiatría. Fundamentos. Madrid, 1973.

Miller, G. A.; Galanter, E., y Pribram, K. H. (1960): Plans and the structure of bebavior. Holt, Rinehart and Winstons, New York, 1960.

MONITZ-RODGON, M. (1976): Single-zyord usage, cognitive development and the beginnings of combinatorial speech. A study of ten English-speaking children. Cambridge University Press. 1976.

MoOre, T. E., y Harris, A. E. (1978): "Language and thought in Piagetian theory". En Siegel, L. S., y Brainero, C. J. (cds.). Alternatives to Piaget. Critical essays on the theory. Academic Press. New York, 1978, I $31-152$.

MOWRER, O. H. (1954): «The psychologist look at language». American Psychologist, 1954, 9, $660-694$.

NiNIO, A., y BRUNER, J. (1978): "The achievement and antecedents of labelling". Journal of Child Language, $1978,5(1), 1-19$.

NOKONY, A. (1978): "Word and gesture usage by an indian child». En LoCK, A. (ed.), 1978, $291-307$.

Oleron, P. (1972): Linguagem e desenvolvimiento mental. Sociocultur. Lisboa, 1978. Original en francés publicado por Pierre Mardaga éditeur. Bruselas, 1972.

Oleron, P., y Herren, H. (196I): «L'acquisition des conservations et le langage. Etude comparative sur des enfants. Sourds et entendants». Enfance, 1961, (14), 203-219.

OISON, D. R. (1970): "Language and thought. Aspects of cognitive theory of semantics». Psychological Review, 1970, 77, 257-273.

Osgood, CH.; Sucl, G. S., y Tannembaum, P. H. (1957): La medida del significado. Editorial Gredos. Madrid, 1976. Original publicado por University of Illinois Press. 1957.

Pérez Pereira, M. (1981): "Piaget y las holofrases». Revista de Psicología General y Aplicada, 1981, 36 (4), 677-696.

Piaget, J. (1946): La formación del símbolo en el niño. F. C. E. México, 1973. Original en francés publicado por Delachaux et Nestlé. Neuchâtel y París, 1946.

Pinget, J. (1954): «El lenguaje y el pensamiento desde el punto de vista genético». En Piaget, J. Seis 


\section{Estudios}

estudios de psicologia, Barral Editores. Barcelona, 1971, I 1 1-124. Original en Revesz, G. (ed.), Thinking and speaking, A Symposium. North Holland Publishing Co., Amsterdam, 1954, 5 I-60.

PiageT, J. (1967): "El lenguaje y las operaciones intelectuales». En PIAGET, J., y otros, Introducción a la psicolinguitstica. Editorial Proteo. Buenos Aires, 1969, 57-75. Original en francés: problémes de psycholinguistique. P. U. F. Paris, 1967.

Piaget, J. (1971): Préface a Ferreiro, E., i971.

RATTAN, M. S. (1974): "The role of language, manipulation and demostraticn in the acquisition, retention and transfer of conservation". The Alberta Journal of Educational Research, 1974, XX (3), 21 7-225.

ReISS, S. (1969): "Two arguments for linguistic behaviorism". Psychological Records, 1969, 19, ,61-572.

SCHI.ESINGER, 1. M. (1971): "Production of utterances and languaje acquisition": Fn Si.oBIN, D. I. (ed.). The ontogenesis of grammar. A theoretical symposium. Academic Press. New York, 1971, 63-10I.

SCHI.ESINGER, I. M. (1974): «Relational concepts underlying language». En Schlesinger, R. L., y Lloyd, L. L. (eds.). Language perspectives: Acquisition, retardation and intervention. Macmillan. Londres, 1974, i $29-151$.

SCHIESINGER, I. M. (1977): "The role of cognitive development and linguistic input in language acquisition". Journal of Child Language, 1977, 4 (2), 153-169.

SCHWAM, E. (1980): "MORE" is "LESS": Sign language comprehension in deaf and hearing children". Journal of Exp. Child Ps., 1980, 29, 249-263.

SINCLAIR DE ZWART, H. (1967): "Adquisición del lenguaje y desarrollo de la mente». Oikos-Tau. Barcelona, 1978. Original: Acquisition du langage et development de la pensée. Dunod. París, 1967.

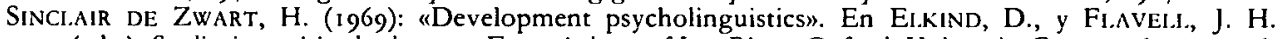
(eds.). Studies in cognitive development. Essays in bonor of Jean Piaget. Oxford, University Press. 1969, 31 5-336.

SINCI.AIR, H. (1971): "Sensorimotor action patterns as a condition for the acquisition of syntax». En Hyxley, R., e Ingram, E. (eds.), 1971 , 1 $21-130$.

SINCLAIR DE ZWART, H. (1973): "Language acquisition and cognitive development. En Moore, T. E. (ed.), I 973, 9-25.

SINCLAIR, H., y FFRREIRO, F. (1970): «Etude génétique de la compréhension, production et répétition des phrases au mode passif. Arcbives de Psychologie, 1970, 40, 1-42.

Skinner, B. F. (1957): Verbal Bebavior. Prentice Hall. Englewood Cliffs., 1957.

SlobiN, D. I. (1971): Introducción a la psicolinguiistica. Paidos. Buenos Aires, 1974. Original en inglés publicado por Scott, Foresman and Company, $197 \mathrm{I}$.

SIOBIN, D. I. (1973): “Cognitive prerequisites for the development of grammar». En FERGUSON, C. A., y Siobin, D. I. (eds.). Studies of Child Language Development. Holt, Rinehart and Winston. New York, $1973,175-208$.

SмIтн, C. S. ( 1980 ): «The acquisition of time talk: relations between child and adult grammars». Journal of Child Language, 1980, 7 (2), $263-278$.

Tsuetkova, L. S. (1977): Reeducación del lenguaje, la lectura y la escritura. Ed. Fontanella. Barcelona, 1977. Original en ruso.

Vygorsky, L. S. (1934): Pensamiento y lenguaje. Comentarios críticos de J. Plaget. La Pléyade. Buenos Aires, 1973. Original publicado en ruso en 1934.

WATSON, J. B. (1929): El conductismo. Ed. Paidos. Buenos Aires, 1972. Original: Behaviorism W. W. Norton and Co. New York, 1925.

Wel.l.S, G. (1974): "Learning to code experience trought language». Journal of Child Language, 1974, 1 (2), 243-269.

WHOPF, B. L. (1956): Lenguaje, pensamiento y realidad. Introducción de J. B. Carroll. Barral Editores. Barcelona, 1971. Original: Language, Thought, and Reality. Editado por J. B. Carroll. M. I. T. Press. 1956.

ZACHRY, W. (1978): "Ordinality and interdependence of representation and language development». Child Development, 1978, 49 (3), 68 1-687.

ZyNkin, N. I. (1976): "Thought and speech". En Prucha, J. (ed.). Soviet studies in language and language behavior. North-Holland Publishing. Amsterdam, 1976, 65-79.

\section{Resumen}

Los modelos tradicionales que pretenden explicar las relaciones entre pensamiento y lenguaje no parecen adecuados, al no poder integrar los datos.obtenidos en las numerosas investigaciones realizadas ultimamente sobre el tema. Se propone un nuevo modelo más integrador, que se inscribe dentro de la linea interaccionista, pero desarrollándola y matizándola.

\section{Summary}

The traditional models trying to explain the relations between thought and language, do not seem to be appropiate as they cannot integrate the data obtained in the different researchs on the 
subject carried out lately. A new model is proposed which is more complete and which is in the interactionist approach, but intro.'reing some developments and variations.

\section{Resumé}

Les modeles traditionels qui prétendent expliquer les rapports entre pensée et langage ne semblent pas justes, car ils ne peuvent pas integrer les donées obtenues dans les nombreuses recherches réalisées dernièrement sur le sujet. On propose un nouveau modèle plus "integrateur», qui s'inscrit dans une ligne interactioniste, pour conquérir un plus grand développement et d'autres nuances. 